\title{
Quality of Experience Tests of an Object-Based Radio Reproduction App on a Mobile Device
}

\author{
ANDREAS SILZLE, ${ }^{1}$ AES Member, REBEKKA SCHMIDT ${ }^{2}$, WERNER BLEISTEINER ${ }^{3}$, \\ (andreas.silzle@iis.fraunhofer.de)
}

NICOLAS EPAIN ${ }^{4}$, AND MARTIN RAGOT ${ }^{4}$

\author{
${ }^{1}$ Fraunhofer IIS, Erlangen, Germany \\ ${ }^{2}$ Fraunhofer SCS, Nürnberg, Germany \\ ${ }^{3}$ Bayerischer Rundfunk, München, Germany \\ ${ }^{4} b<>$ com, Cesson-Sévigné, France
}

\begin{abstract}
Object-based audio (OBA) provides many enhancements and new features. Yet, many of these require the interaction of the user by choosing and selecting the functionalities in visual representations and graphical user interfaces. Basic investigations on the issue of user experience for OBA within the EU research project OPRHEUS identify the necessary criteria and dimensions. The user experience in object-based media comprises three dimensions: audio, information, and usability experience. During the project a radio app for mobile devices was designed, developed, and tested, which includes many of the end-user features available with OBA. A first Quality of Experience (QoE) test to evaluate the radio app was carried out at JOSEPHS, an open innovation lab located in Nuremberg, Germany. The second QoE test took place at $\mathrm{b}<>$ com's user experience lab in Rennes, France. For both investigations, the main objective was to find out how users can access, interact, and appreciate the various new features of OBA. For the first test two typical user and listening scenarios were simulated: mobile listening and at home. As a result, the general acceptance of the new features and functions that come along with OBA is very high. The usability is rated high. Further possibilities for improvements are provided by the test users. The very good perceived sound quality with surround sound over loudspeakers or binaural reproduction over headphones impressed the listeners most, beside all other features. The second test focused mainly on the approach of comparing and evaluating the features from acceptability to acceptance, or from expectations to fulfillment. In the second test, the most appreciated feature was to set fore-to-background balance. This feature was number two in the first test. The importance of speech intelligibility for radio (and TV) is a long known and discussed issue. Now, with OBA and the Next Generation Audio (NGA) codec MPEG-H, solutions are at hand to address it.
\end{abstract}

\section{INTRODUCTION}

"Object-based media is the ultimate approach for creating and deploying interactive, personalized, scalable and immersive content. It allows media objects to be assembled in novel ways to create new and enhanced user experiences, being responsive to user needs as well as environmental and platform-specific factors. Examples are the adaptation of the dialogue level, the flexible playback on any end device or the variable length of a program. This technology is also an essential component for Radio/TV/Online cross-media demands. Moreover, it enables the transition from linear to non-linear and both on-air and on-demand listening, using broadcast and IP technologies." ORPHEUS is a completed
European Commission HORIZON 2020 research project that pioneered the development of an end-to-end objectbased media chain for audio content; for more details see $[1,2]$. Two Quality of Experience (QoE) tests evaluate the object-based radio reproduction app on a mobile device ${ }^{2}$ developed during the project. This app includes a high number of end-user features available with OBA and it serves as demonstrator and for test purposes. Among others, these features are included:

- Reproduction level and position of different objects;

- Loudness and dynamic range control;

\footnotetext{
${ }^{1}$ https://orpheus-audio.eu/
}

${ }^{2}$ https://orpheus-audio.eu/results/ $\rightarrow$ The ORPHEUS App 
- Rendering to different reproduction setups, including binaural headphone reproduction and 3D loudspeaker setups;

- Navigation in sections;

- Transcript of spoken word.

The following two sections cover related work and motivation. The fourth section introduces the used technology. The fifth section describes a working model for QoE assessment in an object-based media eco-system. The sixth section gives an overview about the used technology. Sections seven and eight explain the two evaluations done with the ORPHEUS app. A comparison between the two evaluations finishes up the paper.

\section{RELATED WORK}

Object-based audio (OBA) allows for:

a) Multi-dimensional and multi-lingual features;

b) Novel interactive user experiences and personalized audio content;

c) The delivery of acoustic environments in a format agnostic manner, e.g., the same audio content can be delivered to mono, stereo, binaural, five channel surround sound, as well as advanced multichannel and immersive audio systems.

A general introduction to this concept is given in [3]. A Next Generation Audio codec (NGA) is necessary to transport all the required metadata. In the decoder of such an NGA codec the necessary features have to be implemented to provide all these properties. How this is done with the new MPEG-H standard is described in [4]. MPEG$\mathrm{H}$ has been implemented throughout the ORPHEUS project and investigated iOS app. The "Opportunities for Improved Listening Experience in Increased Listener Involvement" with MPEG-H are explained here [5]. A further article about "Curation, responsiveness and user experience" [6] explains further features of OBA and discusses on the possible relation between some of the features of OBA and the expected user experience.

One traditionally extensively evaluated issue of audio codecs is the so-called Basic Audio Quality, often perceptually measured in comparison to the non-coded audio, e.g., by the MUSHRA method [7, 8]. Beyond this, a comparison of hedonic scales for perceptual evaluation (with and without reference) is presented in [9]. The Basic Audio Quality is extended and compared to the Overall Listening Experience or more general to the Quality of Experience in [10]. A further step forward in introducing the testee more actively in the test design is described and tested in "Adjustment/Satisfaction Test for the Evaluation of Personalization in Broadcast Services and Its Application to Dialog Enhancement" [11].

Here the chosen method for the first reported evaluation uses an even more dialog orientated and interactive end-user evaluation for different dimensions of experience.

\section{MOTIVATION}

A continuously and stringent object-based broadcasting approach offers advanced possibilities to create and implement novel ways of encompassing experience and usability features. With audio becoming interactive-within predefined producer authorized limitations-new challenges are opening up to make object-based media features accessible, understandable, and operable. This applies throughout all stages of the media chain during production, distribution, and reception.

In addition, it is not just the audio itself that matters but also the additional services and features (e.g., transcripts, additional text-based information or still pictures) becoming integrative components in the assessment of the media experience.

This challenges the development of appropriate user interfaces in order to make human interaction to control and adjust complex technical metadata and parameters delivered alongside the audio on the different devices appropriate and convenient. Only if this can be achieved, will the user appreciate object-based media technology as an exciting and satisfying experience. As a consequence, domains for examining and evaluating quality of end user experience that were previously evaluated separately will now have to be considered convergent and inclusive.

\section{USED TECHNOLOGY}

One of the main goals of the EU project ORPHEUS was to develop, implement, and evaluate an end-to-end objectbased broadcast chain. For this purpose, a dedicated reference architecture was developed, see Fig. 1, and then published as an EBU tech report [12]. The ORPHEUS iOS app (marked red in Fig. 1) was especially designed and developed to deliver the typical object-based features described in Secs. 1 and 2. Therefore, it also became the central playback device for the Quality of Experience tests.

A basic description of MPEG-H, the applied NGA audio codec, is given in [4]. Fig. 2 depicts the top-level block diagram of the decoder. The test items (described in detail in Sec. 6.1.4), make use of a variety of combinations of audio channels and objects, as well as both usage scenarios, loudspeaker and binaural headphone, were applied for reproduction. More details about the technical implementation of MPEG-H is given in [13] especially regarding dynamic range and loudness control. The channels, sometimes referred to as "channel bed," keep the continuation to the traditional, static way of audio production and reproduction. Audio objects, however, offer more new interactive possibilities - on the production side and for the end-user. The necessary metadata for these audio objects are generated in new production tools, as explained, e.g., in [14], defining their properties and to set the degrees of interactive possibilities for the end-user. This process is explained in [15]. Fig. 3 shows the structure of an example MPEG-H scene as "Audio Scene Information," defining scene components and language selection interactivity. 


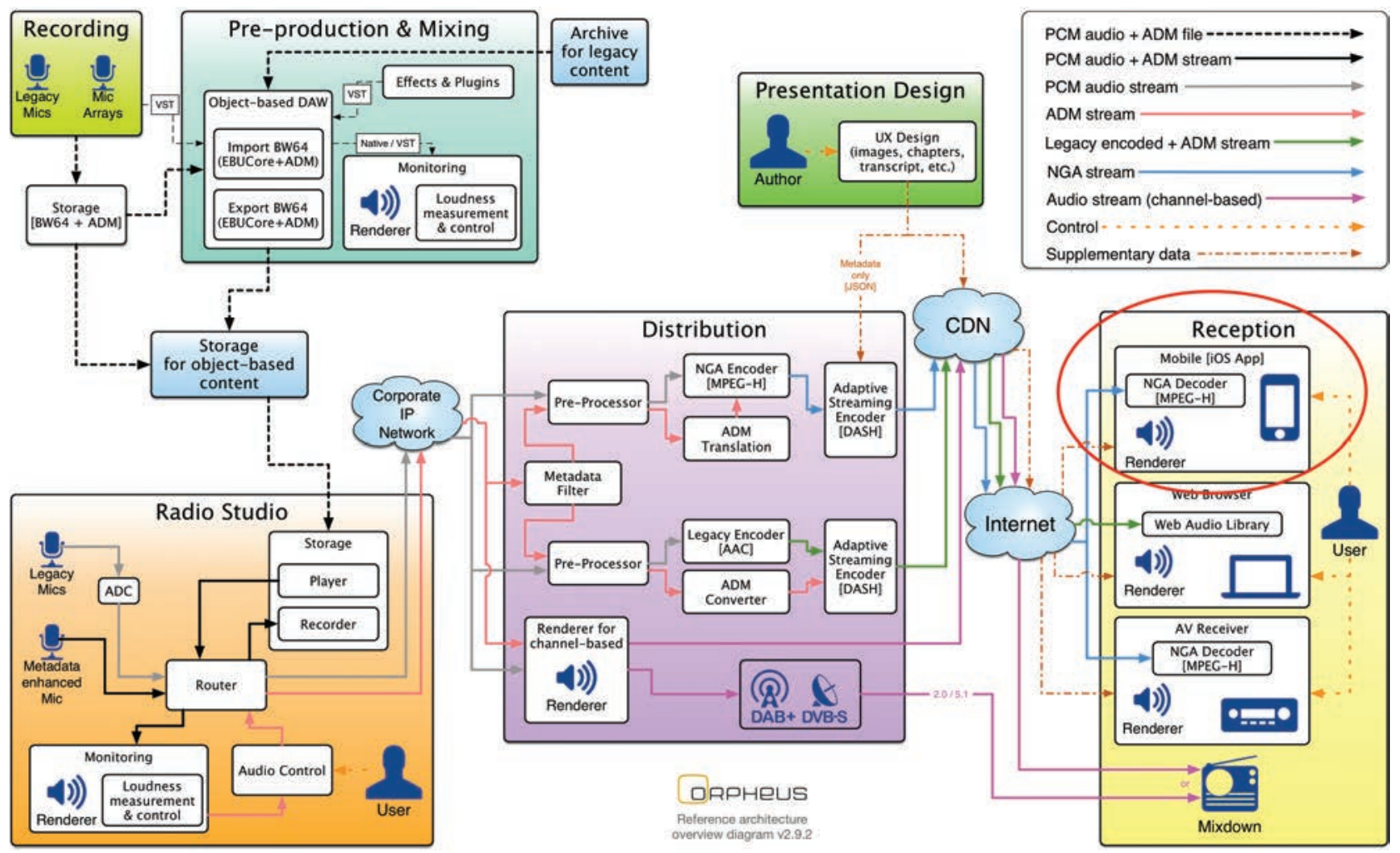

Fig. 1. ORPHEUS reference architecture, [12]. The tested iOS app is marked red.



Fig. 2. Top-level block diagram of MPEG-H 3D Audio decoder as used in the study with dynamic range control (DRC)

Audio objects are processed in the Object Renderer in the decoder with a generalized vector-base amplitude panning (VBAP) [16] algorithm defined in MPEG-H. In case a down-mix of the channels is necessary, the active Format Converter avoids spectral artifacts and signal cancellations. With this structure, the number of transmitted channels and objects are independent from the number of loudspeakers used at the end-user. This is called reproduction setup agnostic [4].

More technical details about the selection and definition of the selected metadata formats and their usage throughout the project is given in [17]. The editing and mixing is explained in $[18,19]$. The requirements on the used formats for audio and metadata are explained in [20]. The implementation experience gained during the project, especially for the Audio Definition Model (ADM) and the metadata formats for non-linear reproduction are described in [21,

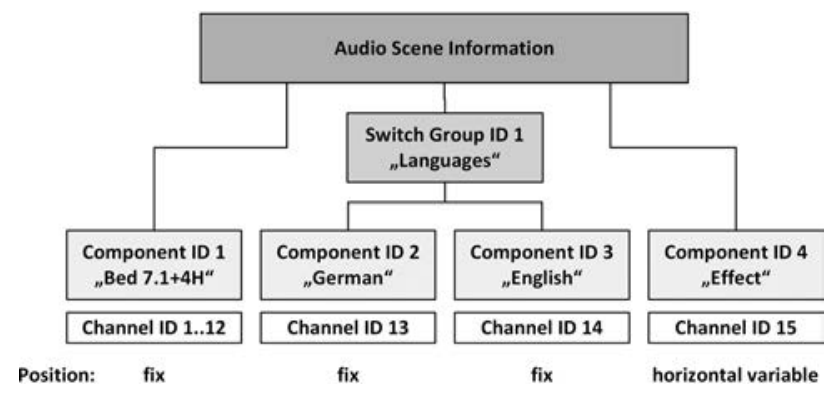

Fig. 3. Example for an MPEG-H audio scene, item "Herbst" (autumn) from Sec. 6.1.4. Channel 1-12: bed, channel 13 and 14: language German and English within a switch group, channel 15: effect channel (bee)

22]. Another outcome of the project was the definition of the "ADM renderer for use in next generation audio broadcasting," EBU Tech document 3388 [23].

\section{DEFINITION OF A WORKING MODEL FOR A QUALITY OF EXPERIENCE ASSESSMENT IN AN OBJECT-BASED MEDIA ECO-SYSTEM}

In the course of the ORPHEUS project, user requests for OBA broadcasting were collected. For this purpose, in-house workshops with creative and technical staff were conducted, to find out what kind of features and requirements are to be considered as necessary or desirable for NGA production and distribution. 

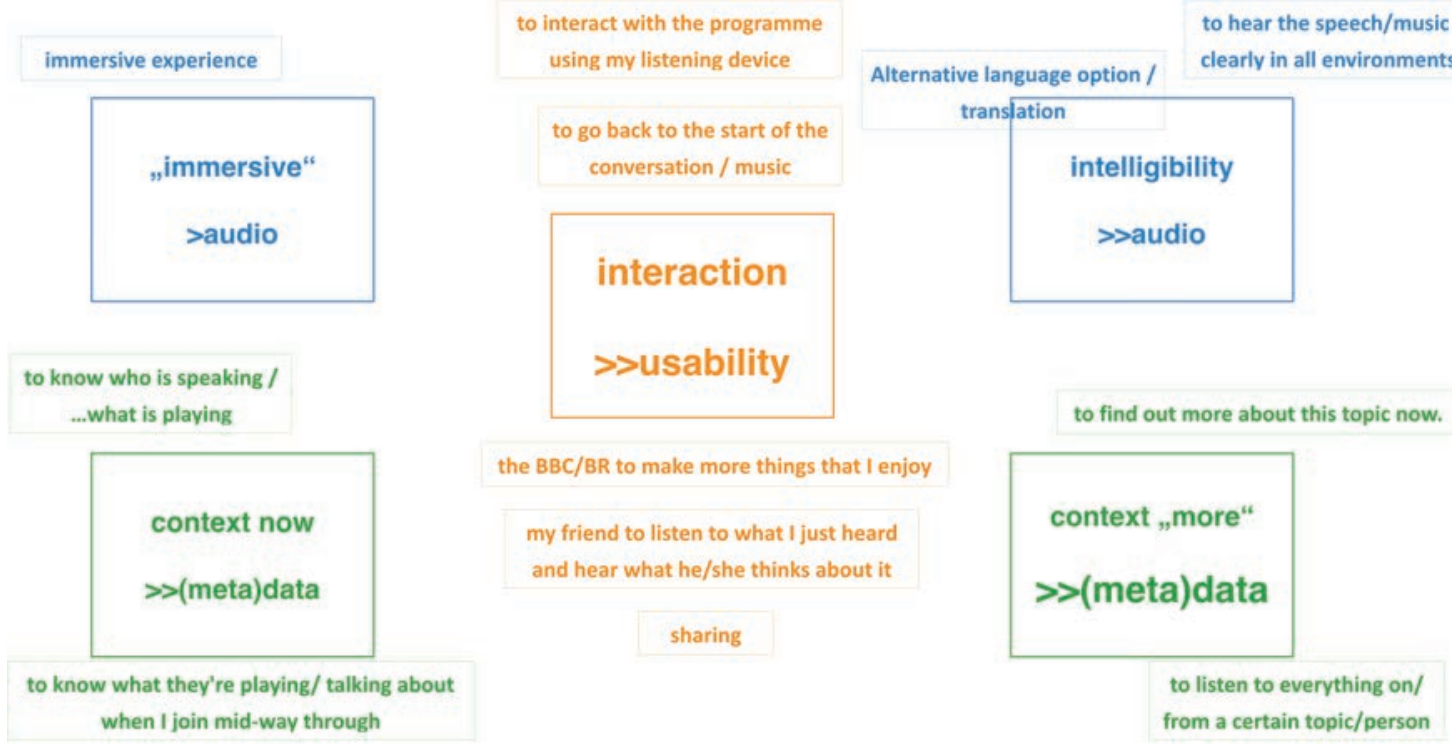

Fig. 4. Identified user requests for object-based audio [25]. The audio experience dimension is presented in blue, the information experience in green, and the usability experience in orange.

At the German public broadcaster $\mathrm{BR}^{3}$, there was a two-day workshop on June 1 and 2, 2016, with 70 participants on the first day and 36 participants on the second day, in order to form 6 small groups for design thinking sprints. The registration and participation was open to all BR staff.

Design thinking [24] is a wide-spread bottom-up methodology for creating and developing software and services involving users at the very initial stage. Its basic principle is to ask them about ideas and wishes. This leads to spontaneous and qualified answers. The material (user requirements and desires) was eventually used to develop the working model for quality of experience assessment in an object-based media eco-system derived mainly from the workshop conducted.

The implications of the basic requests from the first day were enriched in brainstorming sessions on the second day, finding applications and more detailed descriptions for practical scenarios. In a second step, their relation and relevance to existing typical broadcast genres and formats was discussed. At this stage, the implications for production and distribution workflows under the predictable change of broadcast infrastructure were also considered; for details see [25].

In the workshop at the BBC on June 12, 2017, 57 experts participated-32 guests, plus the ORPHEUS and BBC people. The guests were individually chosen because they represent the leaders across Europe in broadcasting, broadcast technology, and broadcast equipment manufacturing. It was based upon the basic question: "What should a future broadcast system enable and deliver to you for a better experience?" This created a full catalogue of wishes. Through discussions within the EU research project, they were clustered and assigned to hypernyms (e.g., intelligi-

\footnotetext{
${ }^{3}$ https://www.br.de
}

bility, immersion) and again assigned to joint hypernyms (e.g., audio, metadata).

The collected und summarized user requests are visualized in Fig. 4. Through this approach, the colloquially uttered basic requests could more clearly be related and assigned to the key-features of object-based broadcasting:
- Interactivity
- Immersion
- Personalization
- Accessibility

A basic approach for practical examination and evaluation of user experience within an object-based media ecosystem as a holistic model based upon the user requests and use cases was developed. The working model is based on three questions:

- Which quality?

- What experience?

$\circ$ Who is the end user?

More details can be found in [26].

Finally, in a third step, this leads to the top domains of the model, the three main experience dimensions:

- Audio experience, referring to the key features immersion and intelligibility (personalization);

- Information experience, referring to contextual metadata of content (accessibility);

- Usability experience, referring to the key features of human interaction with the devices and user interfaces.

In order to test the real-world applicability of our approach, we selected a suitable test setting offering low barrier access to general users in the public. 


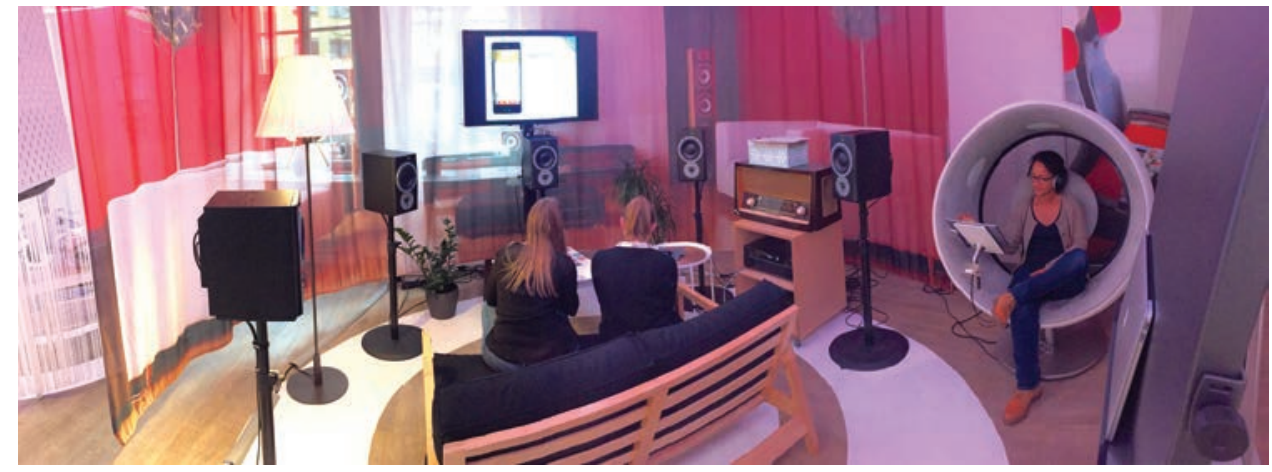

Fig. 5. ORPHEUS test installation at open innovation lab JOSEPHS; left side: loudspeaker reproduction at home; right side: headphone reproduction in a noisy environment (produced by loudspeakers in sonic chair), mobile listening.

\section{JOSEPHS EVALUATION}

\subsection{The Test Concept and Setting at JOSEPHS}

JOSEPHS ${ }^{4}$ is a public open innovation lab or living lab in the city center of Nuremberg (Germany). "Living Lab typically describes a physical test environment in which companies, public authorities and citizens cooperate and test new services, products and technologies" [27]. "Living labs offer a new open innovation platform for companies to engage customers in co-creation and to understand user needs" [28]. "Open innovation simply means opening and diffusing boundaries of corporations and their environments" [29]. New ideas are created from casual conversations, without time limits, between the visitors, also called co-creators, and briefed guides. The methodology applied at JOSEPHS are guided interviews built upon open questions, open feedback, and open dialogue delivering qualitative feedback; see the selected representative participant citations below. To get more quantitative results additional closed questions are asked. These are easier to report; see the result plots below.

A more detailed overview about the tools for collaborating and interacting in living labs is given in [30]. The even more innovative way of co-creation of a service or product in a living lab, as described in [28], is not used in this investigation.

In a preparatory, conceptualization workshop with the experts at JOSEPHS, the setting for the installation based on user stories were shaped. The focus was set on two usage scenarios and aimed to get basic responses on the three defined experience dimensions-audio, information, and usability experience, see Fig. 4, as described in the previous section.

Minimum audio quality evaluation (the first experience dimension) is dependent on the context and the application scenario in which it is investigated $[31,32]$. Therefore, listening experiments and surveys should be conducted in an environment as close as possible to later usage of the enduser of the product, see, e.g., the different results of expert surveys for different auditory virtual environment applica-

\footnotetext{
${ }^{4}$ www.josephs-service-manufaktur.de/en/
}

tions in [33]. Out of this reason, two different main application scenarios for OBA consumption are constructed:

\subsubsection{Mobile Listening}

The listener is sitting in a Sonic Chair ${ }^{5}$, equipped inside with loudspeakers around the head, which reproduce for this mobile listening application a typical flight cabin noise, which was recorded on a real flight. For the playback of the OBA test content in the ORPHEUS iOS app open headphones (Beyerdynamic DT990) are deliberately used in order to get enough outside noise to interfere. For the setup see Fig. 5 right side. The headphone reproduction level is adjusted with the help of the speech containing content material to a natural reproduction level. The level of the masking flight noise is set in a way that the speech content could hardly be understood. The flight noise can be switched on and off by the listener to understand its influence on the content material. For a more detailed test about an adjustment test for dialog enhancement see [11].

\subsubsection{At Home}

The listener is sitting on a couch in the sweet spot of a five-channel surround setup. The loudspeakers are positioned on a circle at the defined azimuth angles $0^{\circ}, \pm 30^{\circ}$, and $\pm 110^{\circ}$ [34]; see Fig. 5 left side. The level of each loudspeaker is measured at the sweet spot and aligned to equal level among all five. An iPad Pro with the ORPHEUS app is connected with an HDMI cable to an AV-Receiver, which is feeding the loudspeakers. The reproduction level can be adjusted by the listener to his or her preferred loudness, dependent on the content, as it is usual in a home environment.

\subsubsection{Technical Setup}

The effort for the setup of these two application scenarios should first arouse the curiosity of the visitors to spend their time on this testing island ${ }^{6}$ in JOSEPHS. It should further simulate during the evaluation the application scenario in a

\footnotetext{
${ }^{5}$ http://www.sonicchair.de/

${ }^{6}$ There were four other islands with other subjects at the same time in JOSEPHS.
} 
plausible and realistic way, having similar listening conditions and optical impressions as in such a real situation. It should evoke the involvement of the participants with the three key elements of the essential characteristics of a living lab: openness, realism, and empowerment [35].

For technical reasons, it was not possible to install a complete 3D loudspeaker system (including elevated loudspeakers) for reproduction of immersive sound in the living room setup, but only a five-channel configuration as shown in Fig. 5. Neutral studio loudspeakers were used in this scenario to be independent from specific preferences or aversions of loudspeaker "sounds" and brands. The tube radio seen in the picture was for decoration only.

No additional EQing or other sound processing was used in the reproduction via headphones or loudspeakers. The output of the OBA stream was directly generated by the MPEG-H decoder and renderer that has been implemented into the ORPHEUS app on the iOS devices. In both scenarios 12-inch iPad Pro devices with a German language surface and additional instructions on what-to-do and howto-use were used. The iOS app is installed in a presentation mode and receives the pilot productions as MPEG-H stream.

\subsubsection{Test Items and Used OBA Features}

The following test items have been installed in the app. The producing partner is given in square brackets:

1. Heute im Stadion: a typical (live) radio report from a football match $[\mathrm{BR}]$

2. Die Kunst des Geräuschemachens (The Art of Foley): radio documentary [BR]

3. Passo Avanti - Mozart Gigue: short jazz adaption of a classical music piece [BR]

4. Erlebe objekt-basiertes Audio (Experience ObjectBased Audio): informative feature [BR]

5. Herbst (Autumn): Poem with 3D soundscape [Fraunhofer IIS]

6. The Turning Forest: cinematic radio drama [BBC]

The items offered the following OBA features:

- Audio experience ${ }^{7}$

- Audio reproduction: mono/stereo/binaural/five channel surround $(1,2,3,4,5,6)$

- Fore-to-background level adjustment $(1,2,4,5,6)$

- Clarity/dynamic range control $(1,2,3,4,5,6)$

- Multi-language (2: DE/EN/FR, 4, 5, 6: DE/EN)

- Audio interaction (3: positioning of the instruments, 5: interactive 3D audio object)

- Information experience

- Text transcript $(1,2,4,5)$

- Additional program information $(1,2,3,4,5,6)$

- Usability experience

\footnotetext{
${ }^{7}$ The numbers in brackets refer to the numeration of the items above.
}

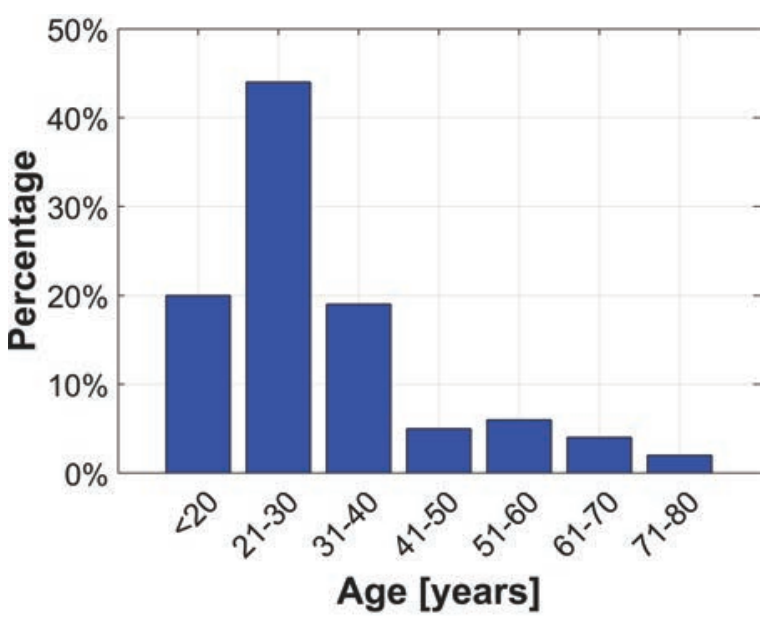

Fig. 6. Age distribution of the 294 participants

Table 1. Number of participants per scenario and age classification

\begin{tabular}{lccc}
\hline & Participants & Age $\leq 30 \mathrm{a}$ & Percentage \\
\hline Airplane scenario & 191 & 108 & $57 \%$ \\
Living room scenario & 202 & 129 & $64 \%$ \\
Both scenarios & 135 & 74 & $55 \%$ \\
\hline
\end{tabular}

- Dial to navigate within the currently playing content $(1,2,3,4,5,6)$

- Points of interest/chapter markers $(1,2,3,4,5,6)$

- Additional pictures (6)

Entering the ORPHEUS area at JOSEPHS, visitors received a short introduction to the specific subject ("audio experience of the future") and, if they were interested in participating, they were asked some basic questions about their listening habits and preferences. After that, the participants were offered to go into one of the usage scenariosmobile listening or at home-and explore the app and the features of OBA in detail. They choose one, the other, or both listening environments.

\subsection{The Findings from JOSEPHS}

In the three months that the experiment ran (Dec. 1, 2017 - Feb. 28, 2018) 294 participants visited the ORPHEUS island and participated in the investigation.

The participants were assigned to one of two age groups: one below and equal to 30 years old and one above, to cut the age distribution about in the middle, see Fig. 6, and with the younger group more used to mobile devices and apps as the older one. The average age of the participants is 31 years. Thirty-six percent of them were female and $64 \%$ male.

Around 200 participants listened to one of the scenarios; 135 of them did both, see Table 1.

First, the participants were asked to select memory cards with the logos of radio programs and streaming apps. This introductory task served to find out more about the subjects' familiarity with present day smartphone applications of broadcasters and other audio service providers in order 


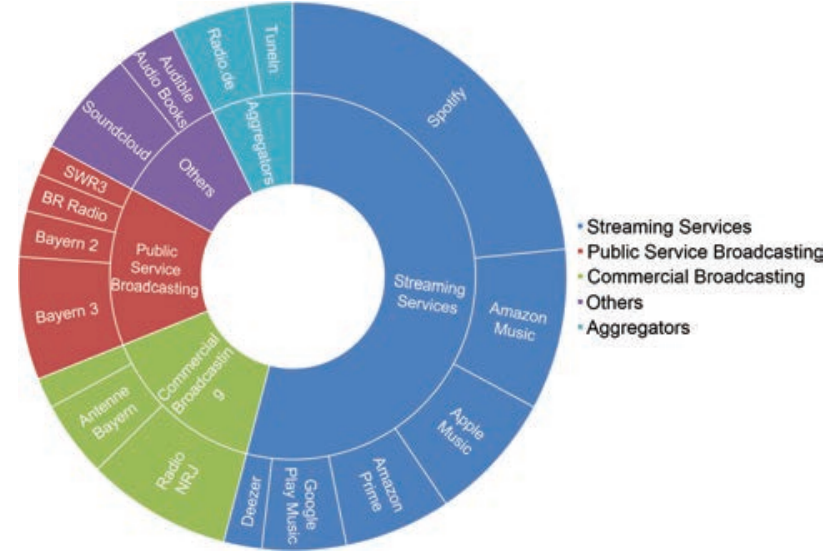

Fig. 7. "Which radio or streaming apps are you using?" $n=242$ (multiple answers are allowed)

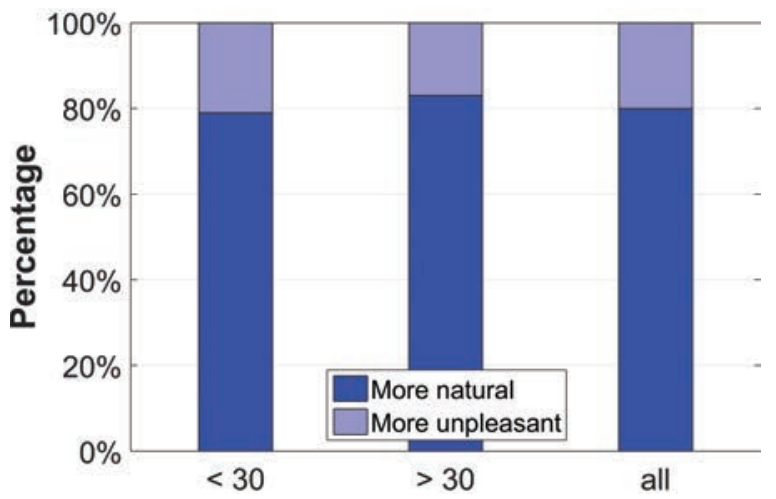

Fig. 8. Is the binaural reproduction more natural than mono or stereo? Answers grouped below $(\mathrm{n}=80)$ and above $(\mathrm{n}=35)$ age of 30 , for airplane scenario.

to categorize them to different user groups. The answers are visualized in Fig. 7. Thirty-eight percent use apps from public service broadcasters, 54\% use streaming services, $43 \%$ of them use Spotify.

\subsection{Audio Experience in the Mobile Listening Airplane Scenario}

In noisy environments, such as inside an airplane, the spoken word is sometimes difficult to understand and quiet parts in music become less audible. The mobile listening setup with headphone reproduction as described above delivers a realistic test scenario.

\subsubsection{Binaural Reproduction}

Participants were asked which reproduction format they liked best. The listeners could switch seamlessly between the formats while listening to different test content. The external airplane noise was switched off at the beginning of the test. A vast majority preferred binaural reproduction $(80 \%)$ compared to stereo $(15 \%)$ and mono $(5 \%)$. The age dependency of the answers is shown Fig. 8.

All questions and answers were induced in conversations between the participants and the JOSEPHS guide. The used guideline for the questions can be found in [36] (in Ger- man). This was only a loose guideline for the conversation, not to break the intention of an Open Innovation Lab by closed lab questions. Not all participants answered every question. Some only with this method achievable comments (in translation) are presented below (more can be found in [36]). The selection was done by one Open Innovation Lab expert (one of the authors), meant as a representative selection. Beside experience, there is no formal method used for this selection.

Some of the positive comments:

"I'm blown away by the binaural sound, really knocked out." (f, 23)

"The sound is especially impressive for football broadcasts." (m, 48)

"Once you've heard binaural, you don't want to hear anything else." ( $m, 37)$

Some of the negative comments:

"Instead of binaural, I would prefer to hear louder ambient sounds." (f, 26)

"The binaural sound gives me a headache." $(\mathrm{m}, 54)$

"The sound is unpleasant. I feel like I'm in a helmet." $(\mathrm{m}, 38)$

For this and the following evaluated OBA features, an equal number of positive and negative comments are presented. The quantitative agreement to the feature is given by the results presented in the figures, not the number of given or presented comments.

Summary and interpretation: The binaural reproduction was vastly preferred. This result is more positive than from many other closed lab binaural listening tests, e.g., [37]. Still, a small percentage of users have different sound reproduction expectations. This is not a problem because these listeners can easily select the standard stereo reproduction instead of binaural reproduction. This is the advantage of object-based production. With this technology, the rendering is done on the reproduction side and the end-user decides in which format he or she is listening to. Compared to the state-of-the-art technology, not only one predefined format, like stereo, is mixed in the studio and sent to the users.

\subsubsection{Fore-to-Background Balance}

With a slider in the app the level balance between the dialog (foreground) and background music or sounds ( $\mathrm{f} / \mathrm{b}$ ) could be adjusted. The slider range was different between the different items, as the producer was setting the min-max values for this item. For some items, at one end of the scale the background only was audible, at the other scale end the dialog only was audible. For other items the range of the dialog was set to $\pm 10 \mathrm{~dB}$. Additionally, the participant could switch the superimposed airplane noise on and off from the Sonic Chair to hear this influence. After testing the $f / b$ balance function under such conditions, they were asked how satisfied they are with this feature. The results are presented in Fig. 9.

Some of the positive comments:

"The individual sound experience is most important to me." (f, 20) 


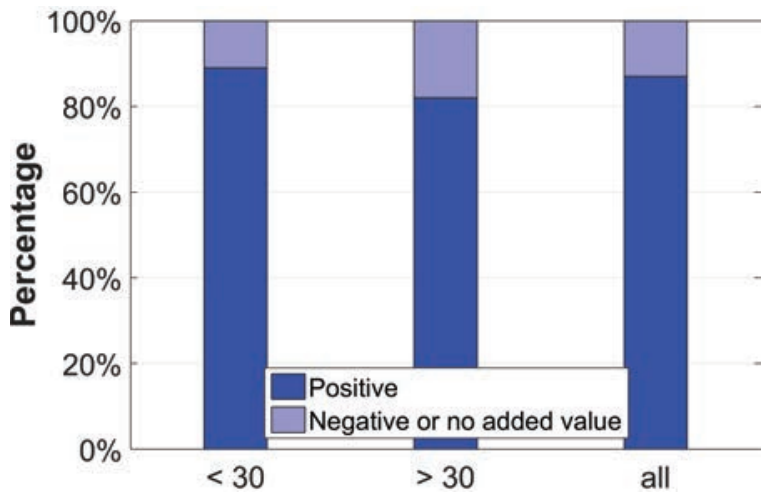

Fig. 9. How is the fore-to-background balance feature? Answers grouped below $(n=75)$ and above $(n=28)$ age of 30 , for airplane scenario.

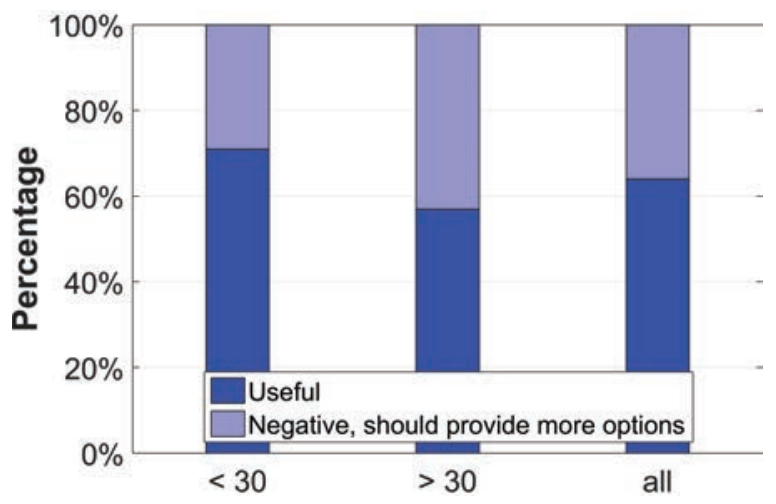

Fig. 10. How useful is the clarity button? Answers grouped below $(n=51)$ and above $(n=23)$ age of 30 , for airplane scenario.

"Finally you can hear the football supporter songs without commentary." (m, 48)

"Background noise no longer impairs listening pleasure." $(\mathrm{m}, 38)$

Some of the negative comments:

"In everyday life I would not use the settings." (f, 23)

"The settings are not yet perfectly worked out." (m, 53)

"I can hardly notice any differences to other sound experiences." (m, 66)

To make this fore-to-background balance even easier to adjust a simple switch was offered in the user interface increasing the foreground by $10 \mathrm{~dB}$ compared to the background. It was labelled "Clarity, make quiet parts louder." Results for clarity or more scientifically expressed intelligibility are shown in Fig. 10. The MPEG-H loudness adjustment feature takes care that the overall loudness of the item is not changed by this adjustment and that the broadcast loudness settings are still applied. For more details see [13].

\section{Some of the positive comments:}

"With this setting I can understand what I usually would barely hear." (m, 28)

"This setting helps you to understand the speaker better. I think that's great." (m, 48)

"I think this setting is very good because you can get more of the atmosphere." (m, 54)

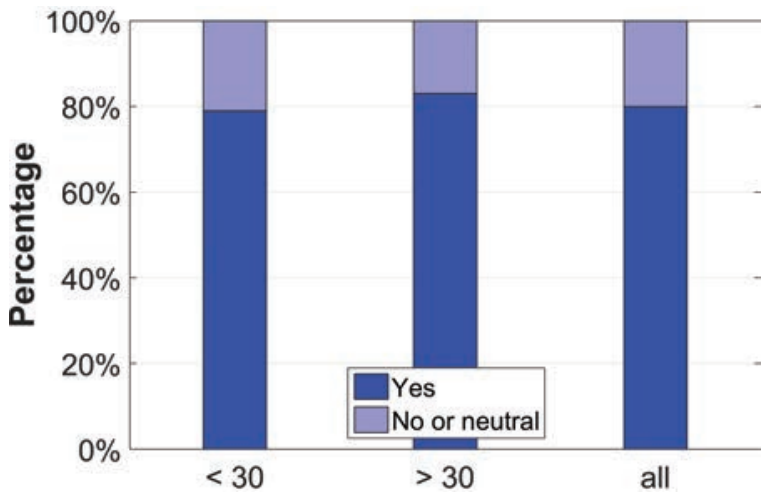

Fig. 11. Do you like surround sound? Answers grouped below $(\mathrm{n}=108)$ and above $(\mathrm{n}=83)$ age of 30 , for living room scenario.

Some of the negative comments:

"I'd rather have just one setting that is perfect for the purpose." (m, 23)

"I don't want to hear every slight noise." (m, 45)

"I want to hear everything as it should be. I don't need this setting." (m, 28)

Summary and interpretation: The new fore-tobackground feature was really appreciated. The adjustable slider was preferred to a simple switch. As an additional feature, the original intended balance setting defined by the sound engineer, tonmeister or producer should be used as default and/or marked on the scale.

\subsection{Living Room Scenario \\ 6.4.1 Audio Experience in the Living Room Scenario "At Home"}

Having been introduced to the setting and selecting one or several items from the ORPHEUS pilot productions and comparing them in various output formats (mono, stereo, five channel surround), listeners were asked: How do you like the five channel surround reproduction and why. Results are presented in Fig. 11.

Some of the positive comments:

"Very cool. You can really feel it." (f, 22)

"I could imagine that in a car." (f, 54)

"I find the feeling of the music around myself very pleasing." (f, 61)

Some of the negative comments:

"I miss the bass." (m, 23)

"The good old stereo sound is sufficient for me." ( $m, 45)$

"I don't like the sound, because I prefer listening to music through headphones." (m, 24)

Summary and interpretation: A large majority of listeners preferred five-channel surround sound. From the comments given, it seems that many people had never really experienced surround sound reproduction in this context, even though it has been on the market for more than 20 years. Other tests have shown even the advantage of 3D reproduction over $2 \mathrm{D}$ reproduction [38], which is possible with the MPEG-H codec. 

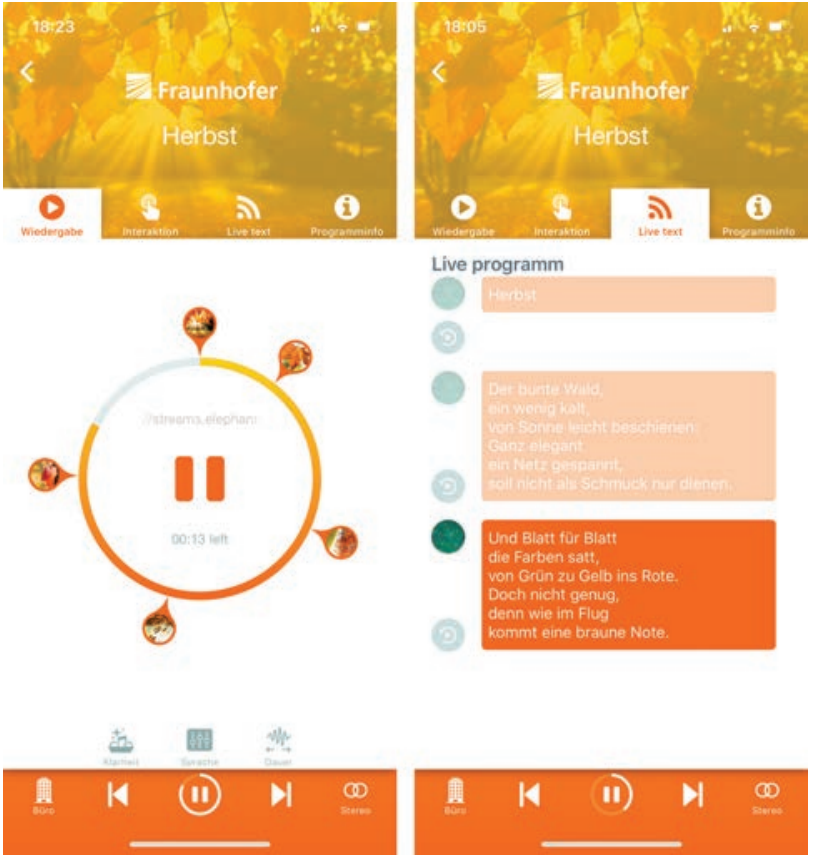

Fig. 12. Two pages of the user interface of the ORPHEUS app. Left side: transport wheel with chapter markers. Right side: transcript of the spoken word.

\subsubsection{Information Experience}

The "live text" transcript presents the spoken language as readable text. Backward and forward navigation can be facilitated by scrolling up and down the text feed (or with transport wheel), as shown in Fig. 12.

Some of the positive comments:

"Useful when I can't turn on my smartphone's sound." (m, 11)

"I can't hear so well anymore. The transcript would make listening to the radio easier for me." $(\mathrm{m}, 60)$

"When my boyfriend interrupts, I can just read it all." (f, 31)

Some of the negative comments:

"I listen to podcasts on the go and don't want to read along." (f, 20)

"If I want to read, I'm reading a book, not a transcript." (f, 58)

"When I read, I read. When I listen, I listen." (m, 24)

The participants were asked how useful text transcript is and why. Results are shown in Fig. 13.

Summary and interpretation: About half of the participants found the text transcript feature useful. Several special cases were mentioned where this feature is useful: repeating it again, people with hearing difficulties, learning a language, translation. Others did not see the benefit of the feature.

\subsubsection{Usability Experience}

In the app, a circle or dial wheel was applied for navigation within an item. It also includes markers for chapters or points of interest and make them directly accessible. Concerning the usability of this concept lis-



Fig. 13. How useful is the transcript? For information experience. Answers grouped below $(\mathrm{n}=86)$ and above $(\mathrm{n}=70)$ age of 30 , answers from both scenarios together.

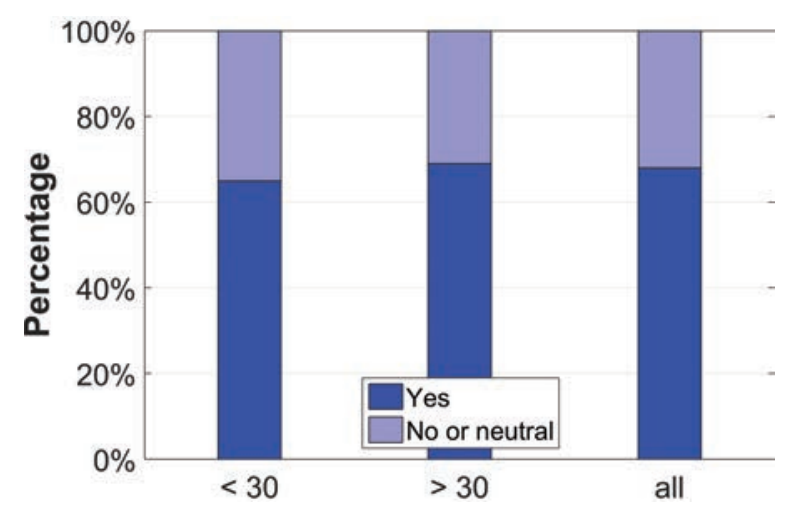

Fig. 14. "Is the usability of transport wheel good?" Answers grouped below $(n=17)$ and above $(n=13)$ age of 30 , answers from both scenarios together.

teners were asked: "How do you like the dial wheel for navigating within the item?" Answers are reported in Fig. 14.

Some of the positive comments:

"I find the design and the operation of the dial attractive." (f, 21)

"If you've missed something, you can rewind intuitively." (m, 28)

One of the negative comments.

"The transport wheel is too small-especially for elderly."

Summary and interpretation: About two third of the users preferred the possibilities of the transport wheel. Regarding the usability questions, the participants, called cocreators in JOSEPHS, responded mainly on the navigation dial. They spontaneously added some more features on a wish list: e.g., search function, a user interface for people with larger fingers, music or program adaptive presets, and user-defined presets. This additional input is an advantage of the open innovation lab method.

At the end of the dialog more general questions are asked.

On the questions "Do you like the new user features?" $65 \%$ answer with yes and $26 \%$ with ok (of $n=120)$. 
On the question "Would you use the app?" $45 \%$ answer with yes and $19 \%$ with maybe (of $\mathrm{n}=196$ ).

This suggests that most people like the new features of the app provided by the object-based approach.

Some of the positive general comments:

- Good binaural sound

- Access to interesting radio programs

Appealing, innovative design

- Individuality, because you can create your own profile

- Intuitive and easy to use

- Use: yes, if free of charge and data protection is guaranteed

Some of the negative general comments:

- Too many functions

- No added value because I am more a casual listener

- The app is not useful because it does not provide presets, you have to set everything yourself ${ }^{8}$

- Cumbersome handling

- Not much different from existing apps

- Use of the app: only if it is integrated in Spotify, otherwise not useful

\subsection{Summary of the JOSEPHS Evaluation}

The majority of the participants listen to music or spoken word programs casually or in a mobile situation. Streaming services, such as Spotify, are the most popular audio apps among the participants. Most of the participants rated binaural sound as better and more natural than mono or stereo.

Most of participants find the possibility to change the fore-to-background balance or "turn up/down the volume of specific sounds, e.g., the announcer" useful. A majority of participants enjoyed the five-channel surround sound loudspeaker reproduction in the living room setup.

About half of the participants would use the transcript. The transcript is regarded mainly useful for repeating something, for people with hearing impairments, for language learning, and translation. Some participants would like an easier navigation and search function as well as more presets within the app. Overall, controls and font sizes should be bigger.

The majority of participants liked the new app features. Overall, audio experience of surround and binaural 3Dheadphone sound are the most popular features; see Fig. 15. Clarity or intelligibility and setting the fore-to-background balance is rated second.

Just over half of the participants would use the app. Others would like to see the features in the apps of the existing audio streaming providers.

The general acceptance of the new features and functions provided by OBA is very high. Much potential for OBA is recognized, but some users propose that us-

\footnotetext{
${ }^{8}$ There were presets but maybe too hidden.
}

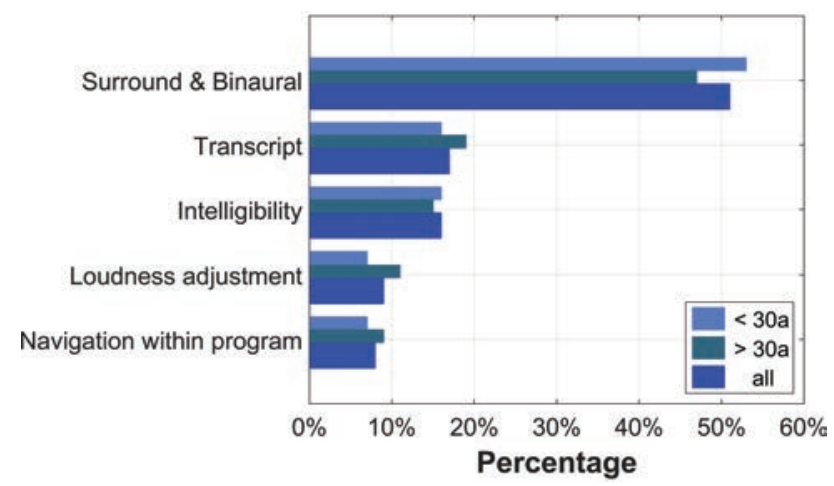

Fig. 15. "Which feature or function do you like most?" Answers below $(n=72)$, above $(n=46)$ age of 30 and all, from both scenarios together.

ability should still be improved. A very positive surprise is that the audio experience with surround sound or binaural reproduction impressed the listeners most. Until now, this aspect of "reality-like listening" was apparently underestimated in audio production and commercial products.

\section{ACCEPTABILITY / ACCEPTANCE EVALUATION}

In parallel to the study conducted at JOSEPHS, the authors conducted a separate evaluation with the same app that took place at the institute of research and technology $\mathrm{b}<>$ com, located in France.

\subsection{Motivation}

The aim of this study was to understand how non-expert users judged the different aspects of the ORPHEUS iOS app and, more generally, Object-Based Audio. One interesting aspect in that regard was to investigate whether the user's perception changed after using the app. Therefore, an acceptability / acceptance approach was taken for this study inspired by that described in [39]. Acceptability refers to prospective judgments on technologies or products before use [40], whereas acceptance refers to judgments on and behavioral reactions to products after use [41]. It is both important to know for a new technology or product: acceptability and acceptance. First, is the user interested to investigate the new product; and second does it fulfill the implementation requirements of the user?

\subsection{Methodology}

The study involved 21 participants and took place from December 2017 to January 2018. It consisted of three main steps, which are described below.

\subsubsection{Initial Questionnaire}

First, the subjects were asked to fill out a questionnaire. The questionnaire in French can be found here [36]. The aim of the questionnaire was to gather information about their eagerness for new technology, their radio listening habits, and their expectations regarding the features offered 
by the ORPHEUS iOS app, and more generally, objectbased audio.

The questionnaire started with a series of questions focusing on the profile of the test participants. Specifically, the participants were asked:

- Their age and gender

- Whether they owned a smartphone and which kind

- About their music and radio listening habits using their smartphone

- About their general view on new technologies

The questionnaire then provided a quick description of the app. "As part of the ORPHEUS project, a new mobile radio application is being developed. In addition to the usual features of a mobile radio application, this one offers several features:

1. Navigating between the chapters of a program

2. Displaying a text transcript of the program

3. Adjusting the "audio clarity" (fore-/background balance)

4. Interacting with the content (moving sound sources or changing the listening perspective)

5. Choosing the program language

6. Setting the rendering format (mono, stereo, or binaural)

7. Adapting the duration of the program."

For each feature, as well as for the iOS app as a whole, the participants were asked to rate on a $0-10$ scale how much they agreed with the following statements:

- This app/feature seems easy to use

- This app/feature seems useful

- This app/feature seems innovative

o It is likely that I will use this app/feature in the future.

\subsubsection{Test of the ORPHEUS iOS App}

After they filled the first questionnaire, the participants were asked to test the ORPHEUS iOS app. This test occurred several days after the initial questionnaire in order to reduce the fatigue of the participants and so that their answers would be more consistent.

In order to test the app, the participants were instructed to perform six tasks, with each task focusing on specific features:

- Chapter navigation - To test this feature the participants were asked to select the program named "Experience Object-Based Audio" and jump from chapter to chapter.

- Text transcript - The participants were instructed to select the "Art of Foley" program and activate the text transcript feature.

- Audio clarity - The participants were asked to select the "Live Football" program and listen to the effect of changing the fore-to-background balance.

- Interaction - The participants were instructed to select the program named "Passo Avanti: Mozart Gigue in $360^{\circ} "$ and try the different versions of this content.

- Multi-language - The participants were instructed to select the "Art of Foley" program and switch the language to English.

- Audio presets and rendering - The participants were instructed to configure a profile that used binaural rendering.

The test took place in a quiet, empty room. An iPhone 5 and a pair of Bose SoundTrue circum-aural headphones were used to run the app.

\subsubsection{Final Questionnaire}

Immediately after testing the app, the participants were asked to fill out a questionnaire. Similar to the initial questionnaire, this final questionnaire asked the participants to rate, for each feature as well as for the app as a whole, how much they agreed with the following statements:

- This app/feature is easy to use

- This app/feature is useful

- This app/feature is innovative

○ I will use this app/feature in the future.

A total of 21 subjects participated to the evaluation, however only 16 of them tested the app and took the final questionnaire.

\subsection{Results}

\subsubsection{Participant Profile}

Of the 16 participants that filled out both questionnaires, 11 were men and 5 women. Their average age was 28.6 years (with a standard deviation of 5.9 years). All declared that they owned a smartphone, 6 running on iOS and 10 on Android.

Most users reported that they used their smartphone to listen to music, as illustrated in Fig. 16.

On the contrary, most participants reported that they rarely listened to the radio using their smartphone, as shown in Fig. 17.

Last, a majority of participants were enthusiastic about technology and eager to try out new ones, as shown in Fig. 18.

To summarize these results, the test participants were relatively young, frequent smartphone users, and in majority eager concerning new technologies. Relatively few of them had the habit of listening to the radio using their smartphone.

\subsubsection{Overall Acceptance and Acceptability}

We now present the results regarding the global appreciation of the app. Fig. 19 presents the ratings before and after 


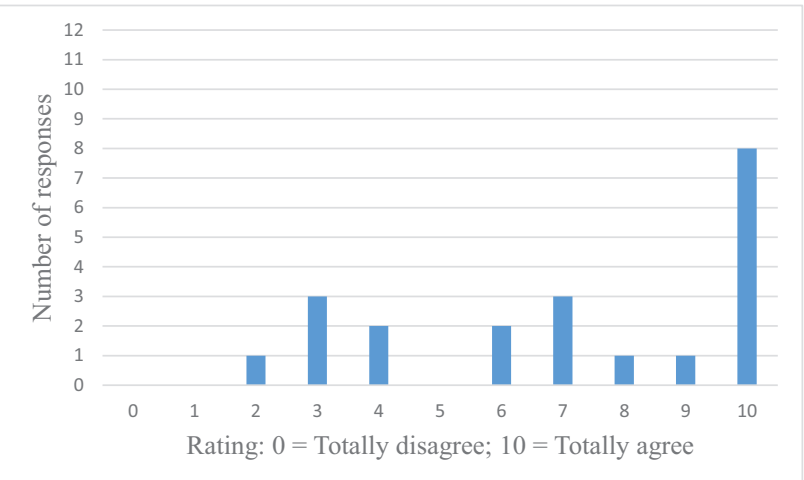

Fig. 16. Responses to the statement "I often listen to music using my smartphone."

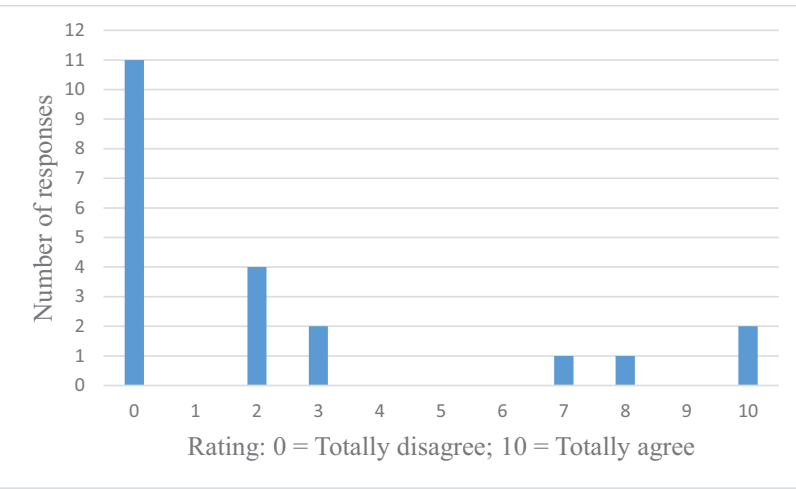

Fig. 17. Responses to the statement "I often listen to the radio using my smartphone."

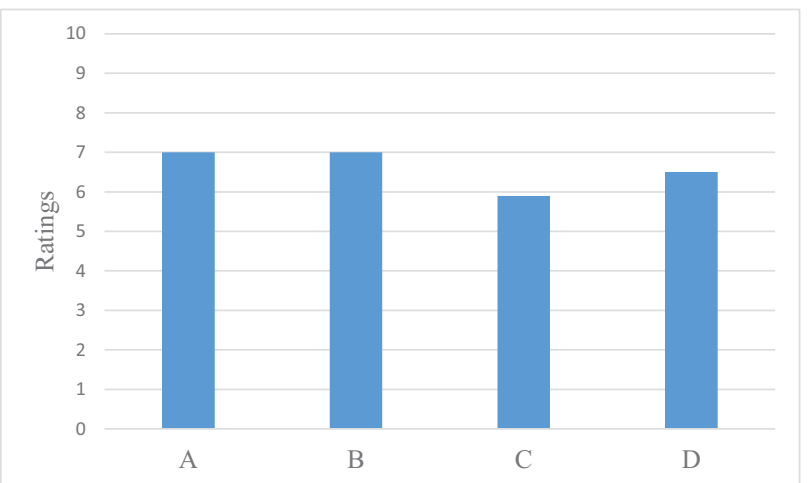

Fig. 18. Responses to the statements: A) "I like to experiment with new technologies"; B) "In general, I am not hesitant to try out new technologies"; C) "When I hear about a new technology I look for ways to try it out"; D) "Among my peers, I am usually the first to try out new technologies."

using the app for the four following criteria: innovation, ease of use, usefulness, intention to use.

The expectations of the participants were globally positive regarding the app. In particular, the app was seen as innovative and expected to be easy to use. However, the participants were dubious about the usefulness of the app and only about half of them thought they would use it in the future.



Fig. 19. Overall appreciation of the ORPHEUS iOS app before use (acceptability) and after use (acceptance).

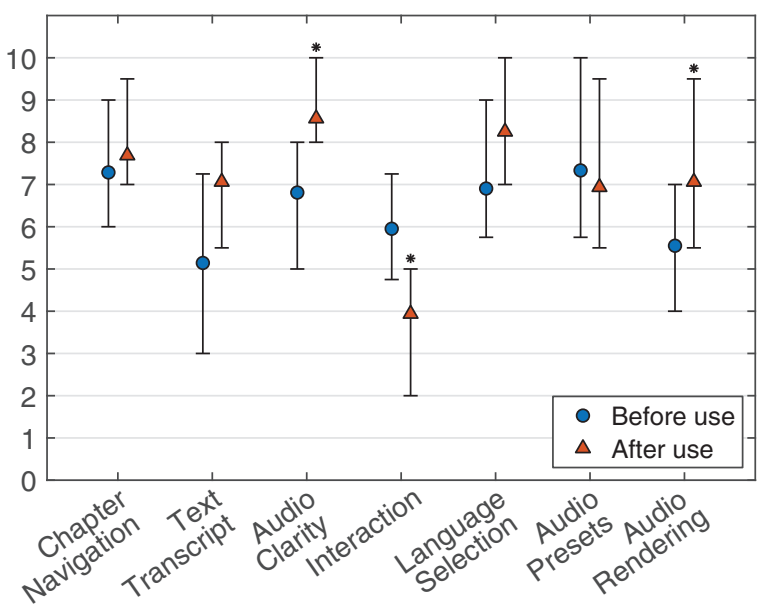

Fig. 20. Perceived usefulness of the different features tested in the iOS app, before and after use. The bars indicate the interquartile range of the user ratings. Note: stars located above the bars indicate that the change in rating was considered statistically significant.

Globally, multi-level regression analysis revealed no significant differences between judgment before and after use $\left(\chi^{2}(1,36)=1.626, p=.020\right)$. Nevertheless, some significant increases can be noted. The increase in ratings is particularly large for the "Ease of Use," $\chi^{2}(1,36)=7.4232$, $\mathrm{p}<.001$ and "Usefulness," $\chi^{2}(1,36)=20.988, \mathrm{p}<.001$ criteria, which indicates that the participants did not expect the app features to be as easy to use and useful as they found during the test (see Table 2 for a complete overview of results). By contrast, the increase in "Intention to Use" is moderate, which is probably related to the fact that a majority of the participants do not use their smartphone to listen to radio programs and do not see themselves doing so in the future. Note that the increase in the "Innovation" rating is also small but the initial rating was already very close to the maximum score.

\subsubsection{Feature Acceptance and Acceptability}

The results regarding the perceived usefulness of the different app features, before and after use, are shown in Fig. 20. Most features were judged more useful after use than before by the participants. The increase in perceived usefulness was significant for the "Audio Clarity" 
Table 2. Results of comparison between acceptability and acceptance by features (items in bold represent significant differences)

\begin{tabular}{|c|c|c|c|c|c|c|c|}
\hline & & \multicolumn{2}{|c|}{ Before use } & \multicolumn{2}{|c|}{ After use } & \multicolumn{2}{|c|}{ Results } \\
\hline & & M & $\mathrm{SD}$ & M & $\mathrm{SD}$ & $x^{2}(1,36)$ & p-value \\
\hline \multirow[t]{4}{*}{ Chapter Navigation } & Usefulness & 7.29 & 2.17 & 7.69 & 1.96 & 3.138 & .0765 \\
\hline & Intention to use & 7.14 & 2.46 & 7.06 & 1.95 & .025 & .8745 \\
\hline & Ease of use & 7.76 & 1.45 & 5.94 & 3.11 & 5.551 & .0185 \\
\hline & Innovation & 6.10 & 2.23 & 5.88 & 2.78 & 8.757 & $<.001$ \\
\hline \multirow[t]{4}{*}{ Text Transcript } & Usefulness & 5.14 & 2.9 & 7.06 & 1.98 & 2.507 & .1133 \\
\hline & Intention to use & 4.76 & 3.00 & 7.06 & 2.08 & 7.607 & $<.001$ \\
\hline & Ease of use & 7.95 & 1.43 & 8.63 & 2.22 & 1.599 & .2061 \\
\hline & Innovation & 6.14 & 2.46 & 6.69 & 3.38 & 1.550 & .2131 \\
\hline \multirow[t]{4}{*}{ Audio Clarity } & Usefulness & 6.81 & 1.89 & 8.56 & 2.13 & 15.479 & $<.0001$ \\
\hline & Intention to use & 6.52 & 2.38 & 8.25 & 1.98 & 6.846 & $<.001$ \\
\hline & Ease of use & 6.62 & 1.12 & 9.19 & 1.11 & 32.130 & $<.0001$ \\
\hline & Innovation & 8.1 & 2.14 & 9.06 & 1.24 & .456 & .4993 \\
\hline \multirow[t]{4}{*}{ Interaction } & Usefulness & 5.95 & 2.50 & 3.94 & 2.43 & 6.846 & $<.001$ \\
\hline & Intention to use & 6.05 & 2.77 & 4.25 & 2.74 & 6.475 & .0109 \\
\hline & Ease of use & 6.81 & 1.60 & 8.13 & 1.67 & 5.777 & .0162 \\
\hline & Innovation & 8.86 & 1.28 & 7.63 & 1.41 & 39.117 & $<.0001$ \\
\hline \multirow[t]{4}{*}{ Language Selection } & Usefulness & 6.90 & 2.90 & 8.25 & 2.32 & .001 & .9781 \\
\hline & Intention to use & 6.67 & 2.98 & 8.00 & 2.61 & 3.490 & .0617 \\
\hline & Ease of use & 8.14 & 1.31 & 9.25 & 0.93 & 13.722 & $<.0001$ \\
\hline & Innovation & 7.81 & 2.16 & 7.13 & 3.34 & .412 & .5208 \\
\hline \multirow[t]{4}{*}{ Audio Presets } & Usefulness & 7.33 & 2.58 & 6.94 & 2.67 & 1.195 & .2744 \\
\hline & Intention to use & 7.24 & 2.30 & 6.63 & 2.99 & .920 & .3374 \\
\hline & Ease of use & 7.10 & 2.02 & 8.31 & 2.06 & 5.024 & .0250 \\
\hline & Innovation & 7.10 & 2.84 & 6.31 & 3.61 & .003 & .9594 \\
\hline \multirow[t]{4}{*}{ Audio Rendering } & Usefulness & 5.55 & 2.19 & 7.06 & 2.64 & 8.842 & $<.001$ \\
\hline & Intention to use & 5.40 & 2.30 & 7.06 & 2.14 & 5.392 & .0202 \\
\hline & Ease of use & 7.45 & 1.67 & 8.75 & 1.65 & 11.727 & $<.0001$ \\
\hline & Innovation & 7.15 & 2.21 & 7.69 & 2.50 & .013 & .9091 \\
\hline
\end{tabular}

(fore-to-background balance) and "Audio Rendering" (switching from stereo to binaural) features. The two features that were found to be the most useful after use were the "Audio Clarity" and "Multilanguage" features.

On the other hand, the usefulness ratings for the "Interaction" feature (ability to change the perspective in the audio scene or move sound sources) decreased significantly after use. Participants reported that they could not hear the difference between the different versions of the program, which could have been caused by a technical problem in the app or in the content itself.

\subsection{Summary of the $b<>$ com Evaluation}

This study aimed to compare the perception of users before and after use of the ORPHEUS iOS app. This comparison was done both on a global level (global user assessment of the app) and on a feature-specific level.

The overall acceptability of the application was relatively high, which shows that the application was perceived positively by the users. In addition, the users found the app easier to use and more useful after they had a chance to try it. This indicates that the app convinced the users of the advantages of object-based audio. However, the fact that ratings increased significantly after use indicate that it is important for users to experience these aspects themselves. Moreover, the participants' intention to use the app did not increase very much after use, which could be related to the fact that most of them rarely listen to the radio using a smartphone.

In terms of features, the "Audio Clarity" (fore-tobackground balance) was the most popular among users, followed by the "Multilingual" and "Audio Rendering" (ability to listen to a binaural version of the programs) features. Conversely, the "Interaction" feature (spatial changes in the audio scene) was perceived as the least useful but this seems to have been caused by a technical problem.

\section{COMPARISON OF THE TWO EVALUATIONS}

The JOSEPHS evaluation in a publicly accessible venue and a run-time of three months resulted in a high number of participants $(n=294)$ covering all ages and types of users. The audio quality evaluation is less strict than in a 
standard listening test but may represent the judgment of a "normal" user more closely. The open-question investigation approach, with all the verbal comments, give much more information and insight how the participants think about and judge the app. On the other side, there is no easy statistical summation possible.

The more traditional lab evaluation from $\mathrm{b}<>$ com uses the advantage of well-defined conditions and questionnaires with clear quantified results. The effort for such an evaluation is smaller as for the open-question investigation.

Both evaluations supplement each other and came to very similar results for the OBA evaluation:

(1) The acceptance after use of the app is very high.

(2) Listening to a radio program on a separate app is not so popular. The integration of the OBA features in the popular streaming provider apps is more promising.

(3) The audio clarity feature or intelligibility gets the highest or second highest ranking of all features in both tests.

Speech intelligibility for radio (and TV) is very important and a long known issue. Now, with object-based audio implemented in the NGA codec MPEG-H, tools are available to address it.

\section{ACKNOWLEDGMENT}

The authors like to thank all participants in the two investigations and the useful input of the reviewers.

This work was funded in parts from the European Union's Horizon 2020 research and innovation program under grant agreement No. 687645 (ORPHEUS project). The paper reflects only the authors' views. The Commission is not responsible for any use that may be made of the information it contains.

\section{REFERENCES}

[1] A. Silzle, M. Weitnauer, and O. Warusfel, et al., "ORPHEUS Audio Project: Piloting an End-to-end ObjectBased Audio Broadcasting Chain," presented at the International Broadcasting Convention (IBC), Amsterdam, The Netherlands (2017), https://doi.org/10.1049/oapibc. 2017.0022 .

[2] M. Weitnauer and A. Silzle, "The ORPHEUS Project: Building the Workflow for End-to-End ObjectBased Audio Broadcasting," FKT Magazin, no. 1-2, pp. 47-51, (2018 Jan.-Feb.).

[3] A. Silzle, R. Sazdov, and M. Weitnauer, et al., "The EU Project ORPHEUS: Object-Based Broadcasting-For Next Generation Audio Experiences," presented at the 29th Tonmeistertagung - VDT International Convention, Cologne, Germany (2016).

[4] J. Herre, J. Hilpert, and A. Kuntz, et al., "MPEGH 3D Audio-The New Standard for Coding of Immersive Spatial Audio," IEEE J. Selected Topics in Signal Processing-Special Issue on Spatial Audio, vol. 9, no. 5, pp. 770-779 (2015), https://doi.org/10.1109/ JSTSP.2015.2411578.

[5] R. Bleidt, A. Borsum, and H. Fuchs, et al., "ObjectBased Audio: Opportunities for Improved Listening Experience and Increased Listener Involvement," presented at the SMPTE Annual Technical Conference \& Exhibition (2014), https://doi.org/10.5594/M001546.

[6] M. Shotton, F. Melchior, and M. Evans, et al., ObjectBased Broadcasting-Curation, Responsiveness, and User Experience, IBC2014 Conference, Amsterdam, The Netherlands (2014), https://doi.org/10.1049/ib.2014.0038.

[7] ITU-R Recommendation BS.1534-3, "Method for the Subjective Assessment of Intermediate Quality Level of Audio Systems (MUSHRA),'Intern. Telecom Union, Geneva, Switzerland (2015), https://www.itu.int/rec/RREC-BS.1534/en.

[8] J. Liebetrau, F. Nagel, and N. Zacharov, et al., "Revision of Rec. ITU-R BS.1534," presented at the137th Convention of the Audio Engineering Society (2014 Oct.), convention paper 9172.

[9] N. Zacharov, C. P. Volk, and T. StegenborgAndersen, "Comparison of Hedonic and Quality Rating Scales for Perceptual Evaluation of High- and IntermediateQuality Stimuli," presented at the 143rd Convention of the Audio Engineering Society (2017 Oct.), convention paper 9879.

[10] M. Schoeffler, A. Silzle, and J. Herre, "Evaluation of Spatial/3D Audio: Basic Audio Quality Versus Quality of Experience," IEEE J. Selected Topics in Signal Processing, vol. 11, no. 1, pp. 75-88 (2017), https://doi.org/10.1109/ JSTSP.2016.2639325.

[11] M. Torcoli, J. Herre, H. Fuchs, et al., "The Adjustment/Satisfaction Test (A/ST) for the Evaluation of Personalization in Broadcast Services and Its Application to Dialogue Enhancement," IEEE Transactions on Broadcasting, vol. 64, no. 2 (2018), https://doi.org/10.1109/ TBC.2018.2832458.

[12] EBU TR 042, "Example of an End-to-End OBA Broadcast Architecture and Workflow," European Broadcasting Union, Geneva, Switzerland (2018), https://tech. ebu.ch/docs/tech/tech3388.pdf.

[13] R. L. Bleidt, D. Sen, and A. Niedermeier, et al., "Development of the MPEG-H TV Audio System for ATSC 3.0," IEEE Transactions on Broadcasting, vol. 63, no. 1, pp. 202-236 (2017), https://doi.org/10.1109/ TBC.2017.2661258.

[14] Y. Grewe, C. Simon, and U. Scuda, "Producing Next Generation Audio Using the MPEG-H TV Audio System," presented at NAB, Las Vegas, USA (2018).

[15] C. Simon, Y. Grewe, N. Faecks, et al., "Field Tests for Immersive and Interactive Broadcast Audio Production using MPEG-H," Set International J. Broadcast Engineering, (2018), http://dx.doi.org/10.18580/setijbe. 2018.5 .

[16] V. Pulkki, "Localization of Amplitude-Panned Virtual Sources II: Two- and Three-Dimensional Panning," J. Audio Eng. Soc., vol. 49, pp. 753-767 (2001 Sep.).

[17] D3.4, "Implementation and Documentation of a Live Object-based Production Environment," Deliveralbe 
of EU Project ORPHEUS (2016), https://doi.org/10.5281/ zenodo.845346.

[18] D3.5, "Specification and Implementation of Reference Audio Processing for Use in Content Creation and Consumption Based on Novel Broadcast Quality Standards," Deliveralbe of EU Project ORPHEUS (2017), https://doi.org/10.5281/zenodo.845330.

[19] D3.6, "Implementation and Documentation of Object-based Editing and Mixing," Deliveralbe of EU Project ORPHEUS (2017), https://doi.org/10.5281/ zenodo.1144096.

[20] D4.1, "Requirements for Representation, Archiving and Provision of Object-based Audio," Deliveralbe of EU Project ORPHEUS (2016), https://doi.org/ 10.5281/zenodo.845334.

[21] D4.3, "Final Report on the Work on Representation, Archiving and Provision of Object-Based Audio," Deliveralbe of EU Project ORPHEUS (2016), https://doi.org/10.5281/zenodo.1173094.

[22] D5.4, "Implementation and Documentation of User Interfaces Associated with Last Personalisation and Interaction Mock-up Scenarios," Deliveralbe of EU Project ORPHEUS (2018), https://doi.org/10.5281/zenodo.1147669.

[23] EBU Tech 3388, "ADM Renderer for Use in Next Generation Audio Broadcasting," European Broadcasting Union, Geneva, Switzerland (2018), https://tech.ebu.ch/docs/tech/tech3388.pdf.

[24] H. Plattner, H. and C. Meinel, and L. J. Leifer., eds., "Design Thinking: Understand, Improve, Apply," in Understanding Innovation (Springer-Verlag, 2011), https://doi.org/10.1007/978-3-642-13757-0.

[25] D3.1, "Requirements, Designs and Workflows of an Object-based Production Environment," Deliveralbe of EU Project ORPHEUS (2016), https://doi.org/10.5281/ zenodo.844008.

[26] D5.3, "Document on Methodology for Evaluation of User Experience," Deliveralbe of EU Project ORPHEUS (2017), https://doi.org/10.5281/zenodo.846264.

[27] V.-P. Niitamo, S. Kulkki, and M. Eriksson, et al., "State-of-the-Art and Good Practice in the Field of Living Labs," presented at the IEEE International Technology Management Conference (ICE), Milan, Italy (2006), https://doi.org/10.1109/ICE.2006.7477081.

[28] K. Greve, V. Martinez, and J. Jonas, et al., "Facilitating Co-Creation in Living Labs: The JOSEPHS Study," European Academy of Management, Paris (2016).

[29] H. Chesbrough, Open Innovation: Researching a New Paradigm (Oxford University Press, 2008).

[30] M. P. Mengual, J. M. Jonas, and S. Schmitt-Rüth, et al., "Tools for Collaborating and Interacting in Living
Labs-An Exploratory Case Study on JOSEPHS," Service Design Proof of Concept, Milano, Italy (2018).

[31] J. Blauert and U. Jekosch, "Sound-Quality Evaluation-A Multi Layered Problem," Acustica - acta acustica, vol. 83, pp. 747-753 ( 1997).

[32] D. Västfjäll, "Contextual Influences on Sound Quality Evaluation," Acta Acustica united with Acustica, vol. 90, no. 6, pp. 1029-1036 (2004).

[33] A. Silzle, "Quality Taxonomies for Auditory Virtual Environments," presented at the 122nd Convention of the Audio Engineering Society (2007 May), convention paper 6993

[34] ITU-R Recommendation BS.775-3, "Multichannel stereophonic sound system with and without accompanying picture," Intern. Telecom Union, Geneva, Suisse (2012), https://www.itu.int/dms_pubrec/itu-r/rec/bs/RREC-BS.775-3-201208-I!!PDF-E.pdf.

[35] B. Bergvall-Kåreborn, M. Holst, and A. Ståhlbröst, "Concept Design with a Living Lab Approach," Proceedings of the 42nd Hawaii International Conference on System Sciences (2009).

[36] D5.6, "Report on Audio Subjective Tests and User Tests," Deliveralbe of EU Project ORPHEUS (2018). https://doi.org/10.5281/zenodo.1306552.

[37] A. Silzle, B. Neugebauer, and S. George, et al., "Binaural Processing Algorithms: Importance of Clustering Analysis for Preference Tests," presented at the 126th Convention of the Audio Engineering Society (2009 May), convention paper 7728 .

[38] A. Silzle, S. George, and E. Habets, et al., "Investigation on the Quality of 3D Sound Reproduction," International Conference on Spatial Audio (ICSA), Detmold, Germany (2011), https://www.researchgate.net/ publication/259974979_Investigation_on_the_quality_of_ 3D_sound_reproduction.

[39] N. Martin, É. Jamet, and S. Erhel, et al., "From Acceptability to Acceptance: Does Experience with the Product Influence User Initial Representations?" International Conference on Human-Computer Interaction (HCI)Toronto, Canada (2016), https://doi.org/ 10.1007/978-3-319-40548-3_21.

[40] J. Schade and B. Schlag, "Acceptability of Urban Transport Pricing Strategies," Transportation Research Part F: Traffic Psychology and Behaviour, vol. 6, no. 1, pp. 45-61 (2003).

[41] G. Schuitema, L. Steg, and S. Forward, "Explaining Differences in Acceptability before and Acceptance after the Implementation of a Congestion Charge in Stockholm," Transportation Research Part A: Policy and Practice, vol. 44, no. 2, pp. 99-109 (2010). 


\section{THE AUTHORS}

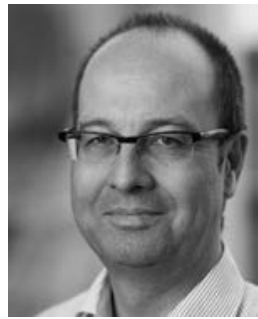

Andreas Silzle

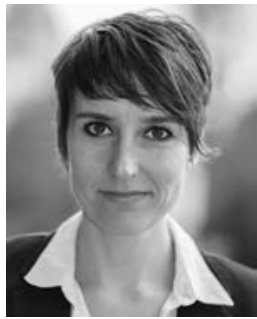

Rebekka Schmidt

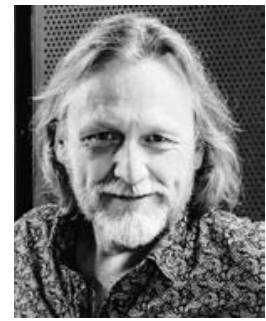

Werner Bleisteiner

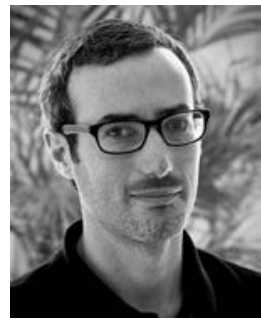

Nicolas Epain

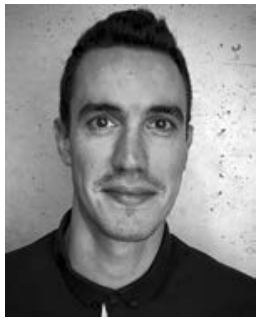

Martin Ragot
Andreas Silzle studied electrical engineering with specialization in acoustics at the University of Karlsruhe, Germany. He worked at the Institut für Rundfunktechnik (IRT) in Munich and for TC Electronic in Denmark. He was a research assistant at the Institut für Kommunikationsakustik (IKA) of Prof. Blauert in Bochum and did his Ph.D. about quality evaluation of auditory virtual environments. Since 2007, he continued at Fraunhofer IIS in Erlangen, where he is a senior scientist. He was actively involved in standardization work in ISO/MPEG and ITU-R. He was the technical coordinator of the EU project ORPHEUS about object-based audio.

Rebekka Schmidt, for four years has been a research associate at Fraunhofer Center for Applied Research on Supply Chain Services SCS and is co-responsible for the open innovation lab JOSEPHS. Prior to her current position, she worked for five years as a project manager in qualitative market research. After her studies of Media Culture at the Bauhaus-University, she gained experience abroad in Madrid and Dublin.

Werner Bleisteiner has $25+$ years of experience in broadcast journalism in radio, television, and Internet. He worked as reporter, author, editor, and producer for various broadcast editorial departments throughout the ARD network.
He has also created numerous radio documentaries on the history and developments of broadcasting and audio technology. Werner is involved in BR's digital radio and media development since 2005. As Creative Technologist he is now designing and coordinating internal and external innovation projects for BR-KLASSIK's Online/Streaming/TV department.

Nicolas Epain obtained his Ph.D. in acoustics from the Université de la Méditerranée (now Aix-Marseille Université) in 2007. From 2007 to 2008 he was a research engineer in the area of 3D audio at Orange Labs, Lannion, France. From 2009 to 2016 he continued his reseach in the area of sound field recording and reproduction at the Computing and Audio Research Laboratory (CARlab) at the University of Sydney, Australia. Since 2016 he has been working as a research engineer for the $\mathrm{b}<>$ com Institute of Research and Technology in Rennes, France.

Martin Ragot is a cognitive psychologist and ergonomist. A former member of the Research Center in Psychology, Cognition and Communication (CRPCC) of Rennes 2 University, France, he is currently working on research projects in cognitive science at the $\mathrm{b}<>$ com Institute of Research and Technology, adopting a user-centered design (UCD) approach. 